\title{
Towards Understanding the Origin of Cosmic-Ray
}

\section{Electrons}

\section{Dimitrii Krasnopevtsev ${ }^{\dagger, *}$}

Massachusetts Institute of Technology, Cambridge, USA

E-mail: Dimitriy.Krasnopevtsev@cern.ch

Precision results on cosmic-ray electrons are presented in the energy range from $0.5 \mathrm{GeV}$ to $1.4 \mathrm{TeV}$ based on 28.1 million electrons collected by the Alpha Magnetic Spectrometer on the International Space Station. In the entire energy range the electron and positron spectra have distinctly different magnitudes and energy dependences. The electron flux exhibits a significant excess starting from 41.2 GeV compared to the lower energy trends, but the nature of this excess is different from the positron flux excess above $25.2 \mathrm{GeV}$. Contrary to the positron flux, which has an exponential energy cutoff of $810 \mathrm{GeV}$, at the $5 \sigma$ level the electron flux does not have an energy cutoff below $1.9 \mathrm{TeV}$. In the entire energy range the electron flux is well described by the sum of two power law components. The different behavior of the cosmic-ray electrons and positrons measured by AMS is clear evidence that most high energy electrons originate from different sources than high energy positrons.

$37^{\text {th }}$ International Cosmic Ray Conference (ICRC 2021)

July 12th - 23rd, 2021

Online - Berlin, Germany

\footnotetext{
${ }^{*}$ Presenter

$\dagger$ On Behalf of the AMS Collaboration.
} 


\section{Introduction}

In this proceeding a precision measurements of primary cosmic-ray electrons up to $1.4 \mathrm{TeV}$ with the Alpha Magnetic Spectrometer (AMS) on the International Space Station are presented. The measurements are based on 28.1 million electron events collected by AMS from May 19, 2011 to November 12, 2017. These results are crucial for providing insights into origins of high energy cosmic-ray electrons and positrons.

The description of the AMS detector is described in detail in Ref. [1] and references therein. The combination of information from the Transition radiation detector, Silicon tracker, and Electromagnetic calorimeter enables the efficient separation of the electron events from background sources. Template fit methods are used to determine the number of electrons in each energy bin. For the analysis details please refer to AMS publication in PRL [2].

\section{Results.}

The latest AMS results on the precision measurements of the electron spectrum together with the GALPROP [3] prediction for the secondary electrons from collision of cosmic rays are shown in Fig. 1 . The spectrum is defined as the flux $\Phi_{e^{-}}$scaled by $\tilde{E}^{3}$, where $\tilde{E}$ is the spectrally weighted mean energy [4]. As seen, the contribution of the collision of cosmic rays to the electron spectrum is negligible in the entire energy range.

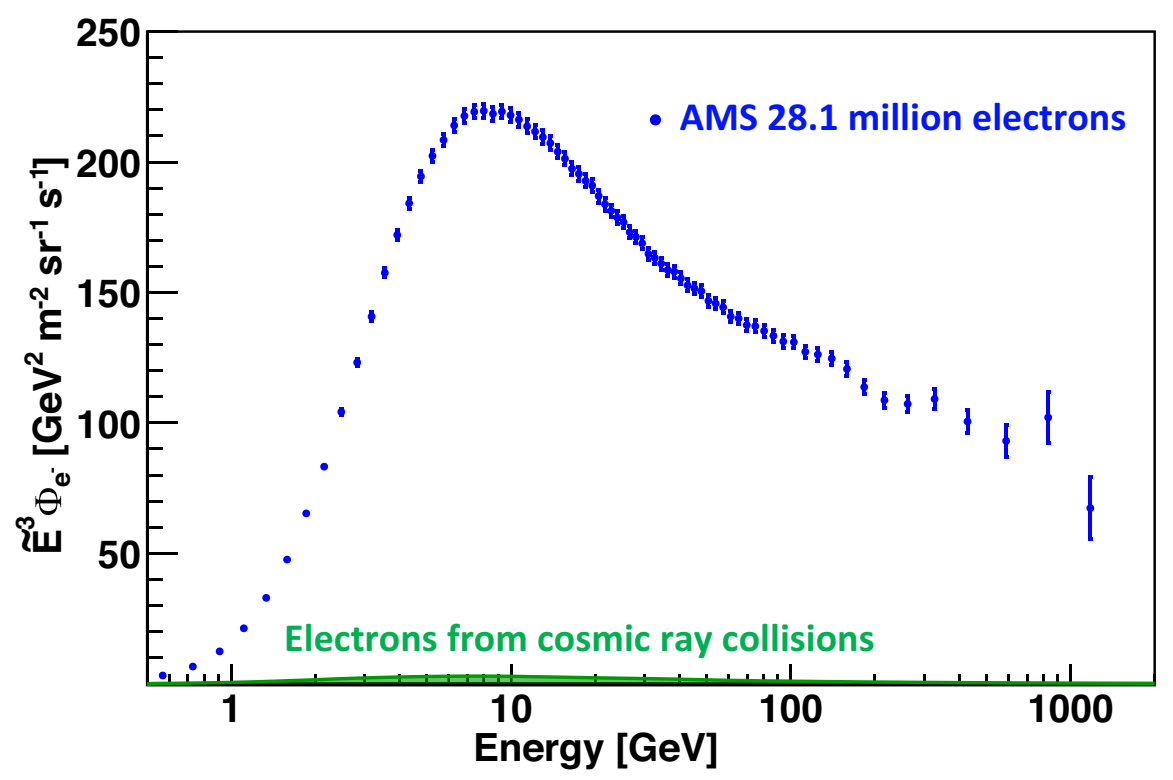

Figure 1: The AMS electron spectrum $\left(\tilde{E}^{3} \Phi_{e^{-}}\right.$, blue data points) together with the GALPROP prediction for the secondary electrons from collision of cosmic rays (green shaded area).

Figure 2 shows the AMS cosmic-ray electron spectrum together with earlier measurements [5-11]. Results from AMS have improved accuracy and extended energy range beyond $1 \mathrm{TeV}$. 




Figure 2: The AMS cosmic-ray electron spectrum. Earlier measurements from PAMELA, FermiLAT, MASS, CAPRICE, AMS-01, and HEAT are also shown.

The changing behavior of the electron flux is examined using the power law approximation, defined in Eq. (1). It is found, the electron flux exhibits a significant excess starting from $42.1_{-5.2}^{+5.4} \mathrm{GeV}$ compared to the lower energy trends. The significance of this change is established at $7 \sigma$. The nature of this excess is different from the positron flux excess above $25.2 \pm 1.8 \mathrm{GeV}$ [12].

$$
\Phi_{e^{-}}(E)= \begin{cases}C(E / 20.04 \mathrm{GeV})^{\gamma}, & E \leq E_{0} \\ C(E / 20.04 \mathrm{GeV})^{\gamma}\left(E / E_{0}\right)^{\Delta \gamma}, & E>E_{0} .\end{cases}
$$

To investigate the energy dependence of the electron flux, the entire energy range is divided into narrow intervals, assuming that the flux behavior follows a power law function in each of these intervals. The flux spectral index, defined in Eq. (2), is calculated over non-overlapping intervals which are chosen to have sufficient sensitivity. The results are presented in Fig. 3 together with the positron results. As seen, the behavior of the electron and positron spectral indices is distinctly different. Electron spectral index hardens starting from $\sim 20 \mathrm{GeV}$ and it is energy independent towards high energy contrary to positron spectral index.

$$
\gamma=d[\log (\Phi)] / d[\log (E)]
$$

The electron flux is fitted with Eq. (3) to check the existence of a finite energy cutoff $E_{s}$. At the $5 \sigma$ level the electron flux does not have an energy cutoff below $1.9 \mathrm{TeV}$, contrary to the positron flux, which has an exponential energy cutoff of $810_{-180}^{+310} \mathrm{GeV}$. These results are presented in Fig. 4.

$$
\Phi_{e^{-}}(E)=C_{S}(E / 41.61 \mathrm{GeV})^{\gamma_{s}} \exp \left(-E / E_{S}\right) .
$$




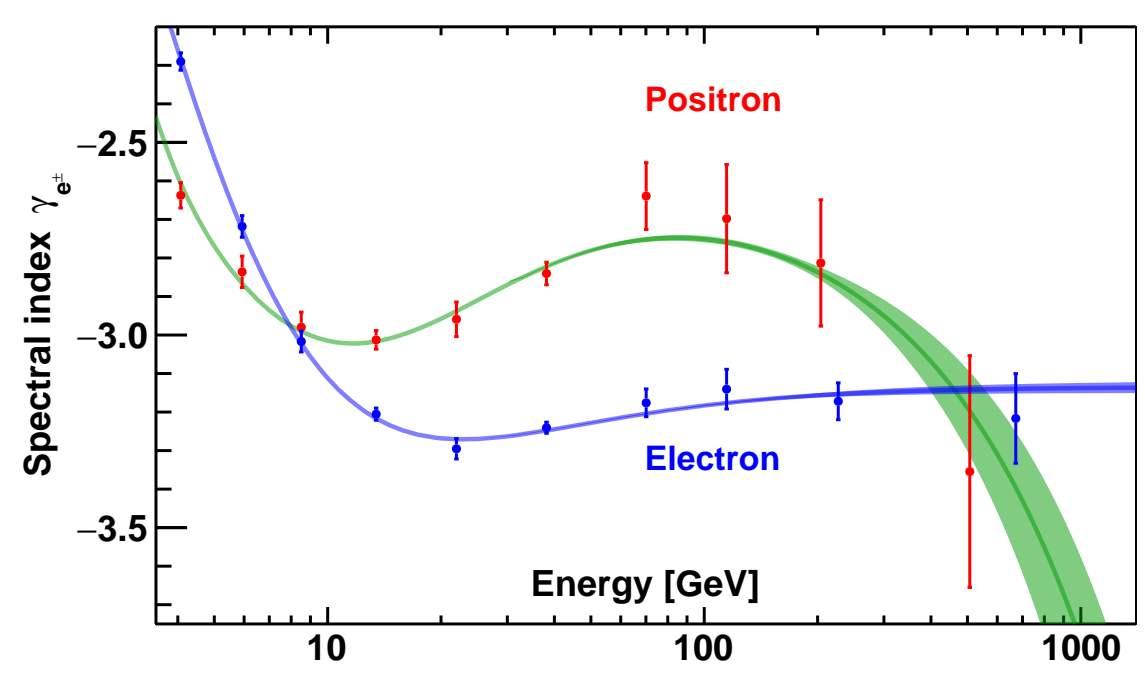

Figure 3: The spectral indices of the electron flux $\Phi_{e^{-}}$and of the positron flux $\Phi_{e^{+}}$as a function of AMS-02 energy. The blue band represents the $68 \%$ C.L. interval of the fit of Eq. (3) to the electron flux. The green band represents the $68 \%$ C.L. interval of a fit to the positron flux (Eq. (4) in Ref. [12]).

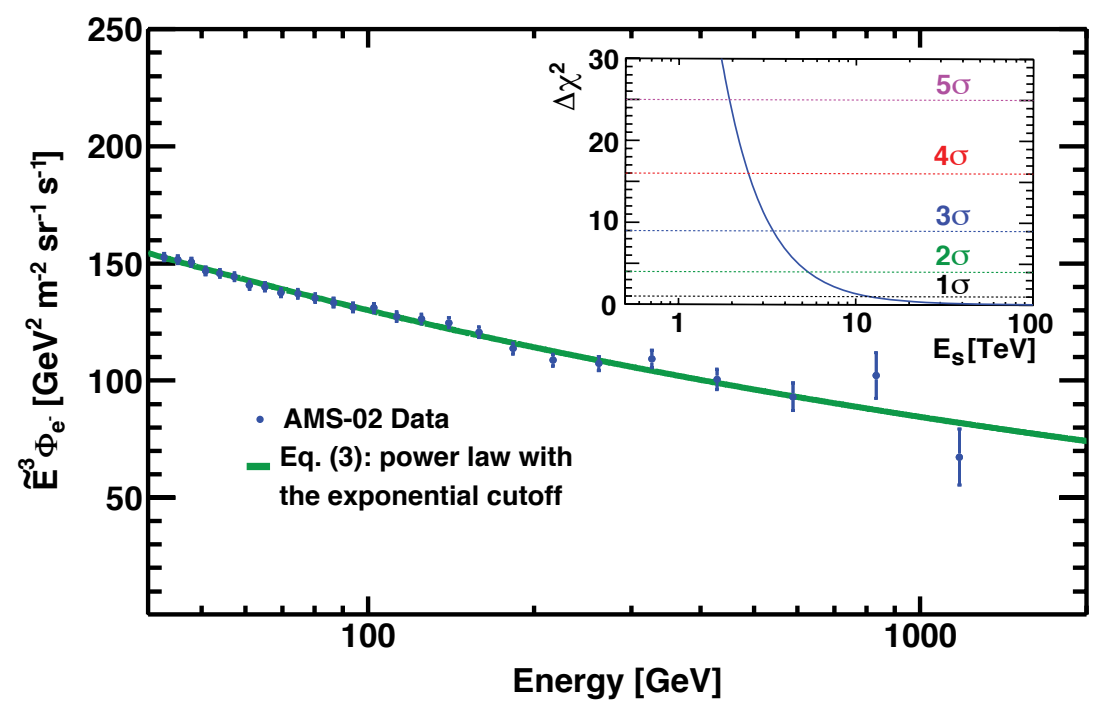

Figure 4: The fit to the electron flux data in the energy range [41.61 - 1400] GeV. The insert shows the study of the significance of the $1 / E_{s}$ measurement by varying all three fit parameters in Eq. 3 to find the minimal $\Delta \chi^{2}$ corresponding to $E_{s}$ values from 1 to $100 \mathrm{TeV}$. The blue curve shows the dependence of $\Delta \chi^{2}$ on $E_{s}$ and the horizontal dashed lines show different significance levels from 1 to $5 \sigma$. 


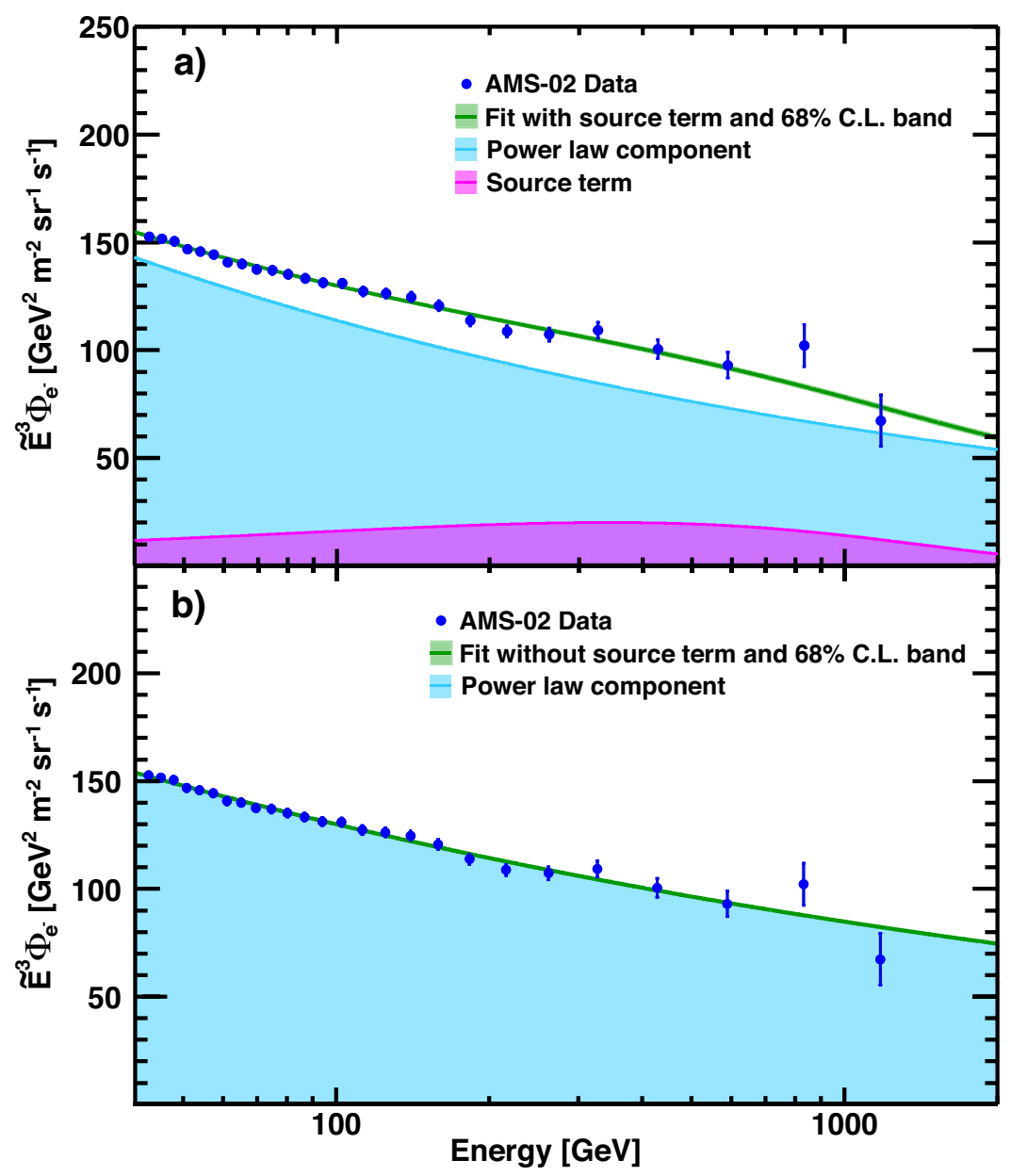

Figure 5: (a) The fit of a power law plus the positron source term $\left(f_{e^{-}}=1\right)$ to the electron flux data in the energy range $[41.61-1400] \mathrm{GeV}$ with the $68 \%$ C.L. (green band), $\Delta \chi^{2} /$ d.o.f. $=15.5 / 24$. The source term contribution, identical to that of positrons, is represented by the magenta area and the power law component by the blue area. (b) The fit of a power law $\left(f_{e^{-}}=0\right)$ to the electron flux data in the energy range [41.61 $1400] \mathrm{GeV}$ with the $68 \%$ C.L. (green band), $\Delta \chi^{2} /$ d.o.f. $=15.2 / 24$. The power law component is represented by the blue area.

New sources of high energy positrons, such as dark matter, may also produce an equal amount of high energy electrons. This hypothesis is tested using the source term from AMS positron analysis [12]. The electron flux is parametrized as a sum of a power law component and the positron source term with the exponential energy cutoff, as defined in Eq.( 4). The result of the fit is presented in Fig. 5a. A similar fit to data, but with $f_{e^{-}}$fixed to 0, yields is presented in Fig. 5b. As seen in figures, the data are consistent both with the charge symmetric positron source term and also with the absence of such a term. Future AMS measurements with improved accuracy and energy reach will reveal detailed features in the electron spectrum.

$$
\Phi_{e^{-}}(E)=C_{e^{-}}\left(E / E_{1}\right)^{\gamma_{e^{-}}}+f_{e^{-}} C_{s}^{e^{+}}\left(E / E_{2}\right)^{\gamma_{s}^{e^{+}}} \exp \left(-E / E_{s}^{e^{+}}\right) .
$$


In addition to a small contribution of secondary electrons produced in the collisions of ordinary cosmic rays with the interstellar medium, shown in Fig. 1, there could be several astrophysical sources of primary cosmic-ray electrons. It is assumed that there are only a few astrophysical sources of high energy electrons in the vicinity of the solar system each making a power law-like contribution to the electron flux $[13,14]$. In addition, there are several physics effects which may introduce some spectral features in the original fluxes $[15,16]$. Therefore, it is important to know the minimal number of distinct power law functions needed to accurately describe the AMS electron flux.

It is found, in the entire energy range $[0.5-1400] \mathrm{GeV}$ the electron flux is well described by the sum of two power law components. The two components, a and b, in Eq. (5) correspond to two power-law functions. The force-field approximation [17] is used to account for solar modulation effect. At low energy, an additional transition term is introduced to account for complex spectral behavior below $\sim 10 \mathrm{GeV}$. A detailed discussion of the parameters and their fitted values can be found in Ref. [2].

$$
\Phi_{e^{-}}(E)=\frac{E^{2}}{\hat{E}^{2}}\left[1+\left(\hat{E} / E_{t}\right)^{\Delta \gamma_{t}}\right]^{-1}\left[C_{a}\left(\hat{E} / E_{a}\right)^{\gamma_{a}}+C_{b}\left(\hat{E} / E_{b}\right)^{\gamma_{b}}\right] .
$$

The fit to the data in the energy range [0.5 - 1400] GeV with Eq. (5) is presented in Fig. 6. As seen, the sum of two power-law functions with the additional transition term provides an excellent description of the data. These functions are very different in shape and in magnitude from those describing the positron flux and indicate that most cosmic-ray electrons originate from different sources than cosmic-ray positrons.

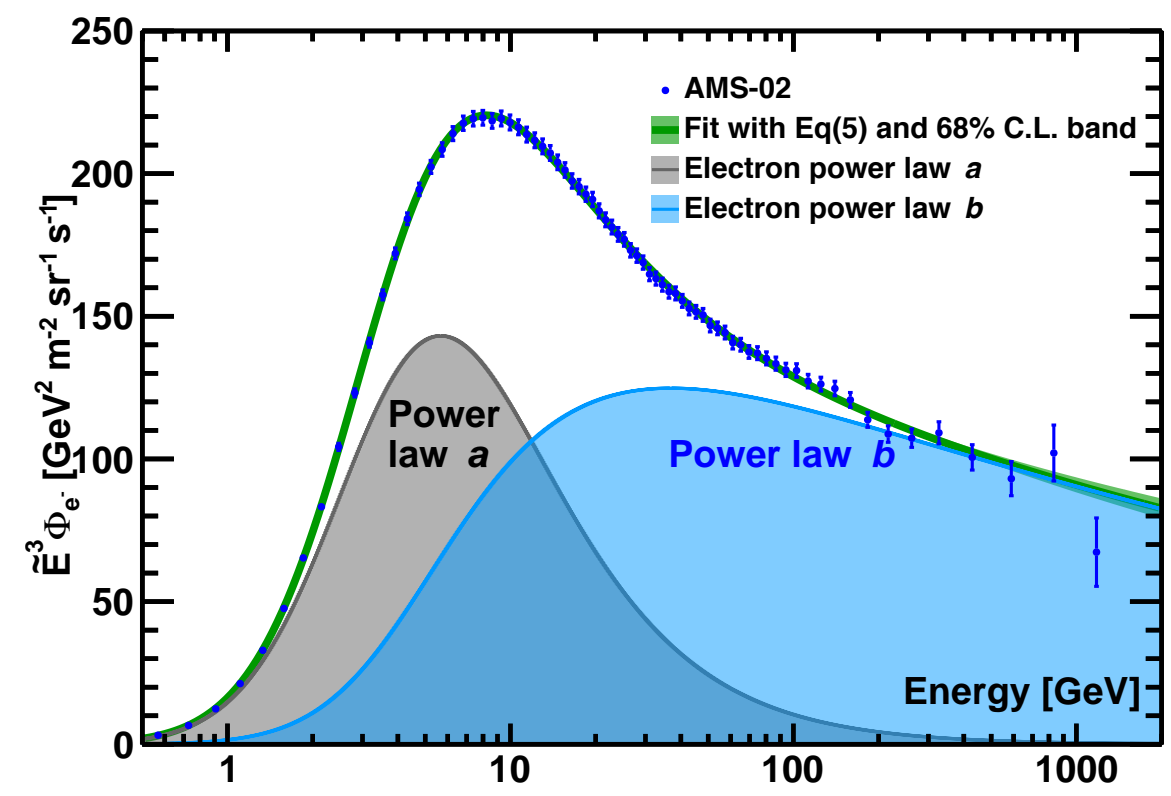

Figure 6: The two power law fit of Eq. (5) to the electron flux data in the energy range [0.5 - 1400] GeV with the $68 \%$ C.L. (green band). The two power law components a and $b$ are represented by the gray and blue areas, respectively. 


\section{Conclusion.}

The high statistics precision measurements of the electron flux from $0.5 \mathrm{GeV}$ to $1.4 \mathrm{TeV}$, based on a data sample of $28.1 \times 10^{6}$ electrons were presented. In the entire energy range the electron and positron spectra have distinctly different magnitudes and energy dependences. The electron flux exhibits a significant excess starting from $42.1_{-5.2}^{+5.4} \mathrm{GeV}$ compared to the lower energy trends, but the nature of this excess is different from the positron flux excess above $25.2 \pm 1.8 \mathrm{GeV}$. Contrary to the positron flux, which has an exponential energy cutoff of $810_{-180}^{+310} \mathrm{GeV}$, at the $5 \sigma$ level the electron flux does not have an energy cutoff below $1.9 \mathrm{TeV}$. In the entire energy range from $0.5 \mathrm{GeV}$ to $1.4 \mathrm{TeV}$ the electron flux is well described by the sum of two power law components. The different behavior of the cosmic-ray electrons and positrons measured by AMS is clear evidence that most high energy electrons originate from different sources than high energy positrons.

\section{Acknowledgments}

This work has been supported by persons and institutions acknowledged in [1,2].

\section{References}

[1] M. Aguilar et al., First Result from the Alpha Magnetic Spectrometer on the International Space Station: Precision Measurement of the Positron Fraction in Primary Cosmic Rays of 0.5-350 GeV, Phys. Rev. Lett. 110141102 (2013).

[2] M. Aguilar et al., Towards Understanding the Origin of Cosmic-Ray Electrons, Phys. Rev. Lett. 122101101 (2019).

[3] I.V. Moskalenko and A.W. Strong, Production and Propagation of Cosmic-Ray Positrons and Electrons, Astrophys. J. 493, 694 (1998).

[4] G.D. Lafferty and T.R. Wyatt, Where to stick your data points: The treatment of measurements within wide bins, NIM A 355, 541 (1995). We have used Eq. (6) with $\tilde{E} \equiv x_{l w}$.

[5] J. Alcaraz et al., Leptons in near earth orbit, Phys. Lett. B 4484, 10 (2000).

[6] O. Adriani et al., Cosmic-Ray Electron Flux Measured by the PAMELA Experiment between 1 and $625 \mathrm{GeV}$, Phys. Rev. Lett. 106, 201101 (2011).

[7] M. Ackermann et al., Measurement of Separate Cosmic-Ray Electron and Positron Spectra with the Fermi Large Area Telescope, Phys. Rev. Lett. 108, 011103 (2012).

[8] C. Grimani et al., Measurement of Separate Cosmic-Ray Electron and Positron Spectra with the Fermi Large Area Telescope, Astron. Astrohys. 1392, 287 (2002).

[9] M. Boezio et al., Measurements of cosmic-ray electrons and positrons by the Wizard/CAPRICE collaboration, Adv. Space Res. 27, 669 (2001). 
[10] M. Aguilar et al., Cosmic-ray positron fraction measurement from 1 to $30 \mathrm{GeV}$ with AMS-01, Phys. Lett. B 646, 145 (2007).

[11] S.W. Barwick et al., The Energy Spectra and Relative Abundances of Electrons and Positrons in the Galactic Cosmic Radiation, Astrophys. J. 498, 779 (1998); M.A. DuVernois et al., Cosmic-Ray Electrons and Positrons from 1 to $100 \mathrm{GeV}$ : Measurements with HEAT and Their Interpretation, Astrophys. J. 559, 296 (2001); J.J. Beatty et al., New Measurement of the Cosmic-Ray Positron Fraction from 5 to 15 GeV, Phys. Rev. Lett. 93, 241102 (2004).

[12] M. Aguilar et al., Towards Understanding the Origin of Cosmic-Ray Positrons, Phys. Rev. Lett. 122, 041102 (2019).

[13] A.M. Hillas, Can diffusive shock acceleration in supernova remnants account for high-energy galactic cosmic rays?, J. Phys. G: Nucl. Part. Phys. 31, R95 (2005); Y.Z. Fan, B. Zhang, and $\mathrm{J}$. Chang, Electron/positron excesses in the cosmic ray spectrum and possible interpretations, Int. J. Mod. Phys. D 19, 2011 (2010).

[14] M. Aguilar et al., The Most Likely Sources of High-Energy Cosmic-Ray Electrons in Supernova Remnants, Astrophys. J. 601, 340 (2004).

[15] L. Stawarz, V. Petrosian, and R. D. Blandford, On the energy spectra of GeV/TeV cosmic ray leptons, Astrophys. J. 710, 236 (2010).

[16] A.W. Strong, E. Orlando, and T.R. Jaffe, The interstellar cosmic-ray electron spectrum from synchrotron radiation and direct measurements, Astron. Astrophys. 534, A54 (2011).

[17] L. Gleeson and W. Axford, Solar Modulation of Galactic Cosmic Rays, Astrophys. J. 154, 1011 (1968). 